\title{
Experimental reproducibility in flow-chamber biofilms
}

\author{
Arne Heydorn, ${ }^{1}$ Bjarne Kjær ErsbøII, ${ }^{2}$ Morten Hentzer, ${ }^{1}$ \\ Matthew R. Parsek, ${ }^{3}$ Michael Givskov ${ }^{1}$ and Søren Molin ${ }^{1}$ \\ Author for correspondence: Søren Molin. Tel: +45 452525 13. Fax: +45 45932809 \\ e-mail:imsm@pop.dtu.dk
}

\footnotetext{
1 Molecular Microbial Ecology Group, Department of Microbiology, The Technical University of Denmark block 301, DK-2800 Lyngby, Denmark

2 Department of Mathematical Modelling The Technical University of Denmark, DK-2800 Lyngby، Denmark

3 Environmental Engineering Group, Department of Civil Engineering, NorthWestern University, Evanston, Illinois, USA
}

\begin{abstract}
The structural organization of microbial communities is influenced by many factors, e.g. nutrient composition, shear stress and temperature. This paper presents a general method for quantitative comparison of biofilm structures and assessment of experimental reproducibility between independent biofilm experiments. By using a novel computer program, cOMSTAT, biofilm structures of Pseudomonas aeruginosa and an isogenic rpos mutant were quantified. The strains were tagged with the green fluorescent protein (GFP) and grown in flow chambers with a defined minimal medium as substrate. Three independent rounds of biofilm experiments were performed and in each round, each of the two variants was grown in two separate channels. Nine image stacks were acquired in each channel $146 \mathrm{~h}$ after inoculation. An analysis of variance model incorporating the factors experiment round, bacterial strain, channel number and image stack number was used to analyse the data calculated by COMSTAT. Experimental reproducibility was verified by estimating the magnitude of the variance of the effects round $\left(\sigma_{R}^{2}\right)$ and the interaction between bacterial strain and round $\left(\sigma_{B R}^{2}\right)$. Mean thickness of the wild-type and rpoS mutant biofilms was estimated at $6.31 \mu \mathrm{m}$ (SE $0.81 \mu \mathrm{m})$ and $16.85 \mu \mathrm{m}$ (SE $0.87 \mu \mathrm{m})$, respectively.
\end{abstract}

Keywords: biofilm structure, quantification, statistical analysis, COMSTAT, reproducibility

\section{INTRODUCTION}

Biofilms are sessile microbial communities growing on surfaces, frequently embedded in a matrix of extracellular polymeric substances. They are characterized by a high population density and by structural organization (Stoodley et al., 1999a). Many different factors have been shown to influence biofilm formation, including the chemical nature of the substratum (Dalton et al., 1994; Cunliffe et al., 1999), the nature of the carbon source (Grotenhuis et al., 1991; Wolfaardt et al., 1994; Møller et al., 1997; Nielsen et al., 2000), carbon source concentration (Wimpenny \& Colasanti, 1997; Picioreanu et al., 1998), osmolarity (O’Toole \& Kolter, 1998), shear stress (Stoodley et al., 1999b; Van Loosdrecht et al., 1995) and population composition (Murga

Abbreviation: GFP, green fluorescent protein. et al., 1995; Kuehn et al., 1998; Lawrence et al., 1991; Nielsen et al., 2000).

Biofilms in nature are often difficult to investigate and experimental conditions are ill defined. Therefore a number of different laboratory-based experimental biofilm model systems have been developed (Palmer, 1999). These systems allow studies of biofilms under defined conditions; such model systems are necessary in order to perform well-controlled reproducible experiments.

There have been various attempts to quantify biofilm structures (see Heydorn et al., 2000, for references). However, despite the many reports on quantification of biofilm structures, the reproducibility of biofilm experiments has not been addressed until now. In this communication we present a general method for quantitative comparison of biofilm structures and assessment of experimental reproducibility between independent biofilm experiments, by using a novel computer program, COMSTAт (Heydorn et al., 2000). 


\section{METHODS}

Bacterial strains and plasmids. The strains used were $P$. aeruginosa PAO1 (Holloway, 1955) and an isogenic rpoS null mutant $P$. aeruginosa MW20 (Whiteley et al., 2000). A $\mathrm{P}_{\mathrm{A1} / 04 / 03}-g f p-\mathrm{T} 0-\mathrm{T} 1$ transposon cassette was inserted into the chromosome of the two strains using pUTtc as delivery plasmid (de Lorenzo et al., 1990) with the cassette cloned in the NotI site. The $\mathrm{P}_{\text {A1/04/03 }}$ promoter, derived from the lac promoter of E. coli DZ291 (Lanzer \& Bujard, 1988), acts as a constitutive promoter in Pseudomonas spp. due to the absence of lac repressor activity (Andersen et al., 1998). By triparental mating between E. coli CC118( $\lambda$ pir) containing the pUT delivery plasmids, E. coli HB101(RK600) acting as a conjugation helper strain, and each of the two recipient strains, the $\mathrm{P}_{\mathrm{A} 1 / 04 / 03^{-}}-g f p-\mathrm{T} 0-\mathrm{T} 1$ cassette was inserted at random positions in the chromosomes of $P$. aeruginosa PAO1 and $P$. aeruginosa MW20. The selected GFP-tagged strains showed no sign of phenotypic changes compared to the parental strains, when tested in liquid medium or flow-chamber biofilms.

Flow-chamber experiments. Biofilms were grown at $30{ }^{\circ} \mathrm{C}$ in three-channel flow cells (Christensen et al., 1999) with individual channel dimensions of $1 \times 4 \times 40 \mathrm{~mm}$ supplied with a flow of $3 \mathrm{ml} \mathrm{h}^{-1}$ of modified FAB medium (Heydorn et al., 2000) supplemented with $0 \cdot 1 \mathrm{mM}$ sodium citrate. The flow system was assembled and prepared as described by Christensen et al. (1999). The substratum consisted of a microscope glass cover slip (Knittel $24 \times 50 \mathrm{~mm}$ st 1 ; Knittel Gläser). Cultures for inoculation of the flow channels were prepared as described by Heydorn et al. (2000).

Image acquisition. Three independent rounds of biofilm experiments were done. In each round, each of the two variants was grown in two separate channels. In each of the four channels nine image stacks were acquired at $146 \mathrm{~h}$ after inoculation. In all experiments, images were acquired from random positions in the upper part of the flow channel, at a distance of 5-10 $\mathrm{mm}$ from the inlet. Images were acquired in the middle two-thirds of the flow channel because the biofilm in the regions near the sides of the flow channel often displayed different behaviour compared to the biofilm in the centre of the channel. Images were acquired at $1 \cdot 0$ to $2 \cdot 0 \mu \mathrm{m}$ intervals down through the biofilm, and therefore the number of images in each stack varied according to the thickness of the biofilm. The nine image stacks covered a total area of $5.625 \times 10^{5} \mu \mathrm{m}^{2}$. Korber et al. (1993) recommended that a minimum area of $1 \times 10^{5} \mu \mathrm{m}^{2}$ be investigated in order to obtain representative data of $P$. fluorescens biofilms. All microscopic observations and image acquisitions were performed on a confocal scanning laser microscope (TCS4D; Leica Lasertechnik). Images were obtained with a $40 \times / 0.75$ air objective. Image scanning was carried out with the $488 \mathrm{~nm}$ laser line from an $\mathrm{Ar} / \mathrm{Kr}$ laser.

\section{RESULTS AND DISCUSSION}

\section{Purpose of reproducibility in biofilm experiments}

In most areas of science, experimental reproducibility is considered fundamental. However, one of the major obstacles in quantification of biofilm structures is the fact that the development of bacterial biofilms is to a certain extent a stochastic process, and independent rounds of biofilm experiments therefore never result in exact structural copies, even if the experimental conditions are kept constant. Despite numerous attempts to

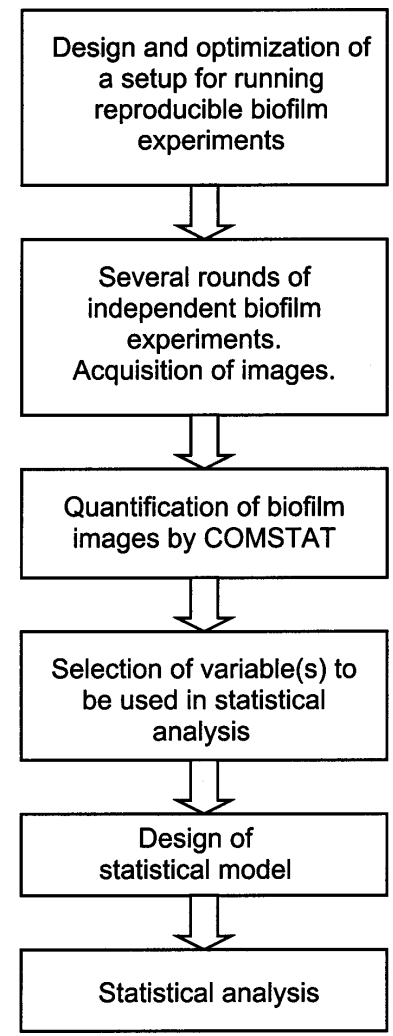

Fig. 1. Steps in quantification and statistical analysis of biofilm structures.

quantify biofilm structures (see Introduction), there have been no attempts to verify the reproducibility of experiments and the statistical significance of results until now. Investigations of biofilms are frequently reported in a descriptive manner, but could advantageously be quantified and checked for reproducibility. Examples where this would apply include (1) analysis of temporal structure development in single-species or community biofilms; (2) impact of specific mutations on biofilm structure; (3) analysis of the influence of changing environmental conditions (e.g. temperature or carbon source) on biofilm structure in single-species or community biofilms; (4) analysis of the influence of antibiotic treatment on biofilm structure. Fig. 1 shows the stepwise process of quantification and statistical analysis of biofilm structures. Below, the steps in Fig. 1 are described in detail.

\section{Designing a setup for running reproducible biofilm experiments}

The most crucial step in Fig. 1 is probably the design of a good model system, because it provides the basis for the entire experiment. A good model system will facilitate the statistical analysis and the interpretation of results, whereas the 'noise' in a poor model system may obscure otherwise observable differences. The design 
and optimization of a setup for running reproducible biofilm experiments includes: (1) minimization of variation between successive rounds of experiments, (2) minimization of variation between channels containing the same strain and (3) minimization of spatial heterogeneity (discussed below) within each channel, while maintaining structural differences between different strains. By identifying and controlling the main factors influencing biofilm formation, such as flow rate, temperature, nutrient composition and the history of the cultures used for inoculation, experimental reproducibility can be improved significantly.

The flow channels used in this study are three-channel flow cells (Christensen et al., 1999) with individual channel dimensions of $1 \times 4 \times 40 \mathrm{~mm}$. The flow channels are machine made from a block of plexiglass. In this way biofilm structure variation caused by small differences in flow channel shape and size is minimized. Since there is a considerable amount of spatial heterogeneity in the flow channels (the biofilm near the inlet is typically thicker than the biofilm near the outlet), the images were acquired at random positions in the upper part of the flow channel at a distance of $5-10 \mathrm{~mm}$ from the inlet. This minimizes structural heterogeneities caused by spatial differences in the flow channels.

Biofilms were grown on a chemically defined medium with citrate as the only carbon source. An important factor turned out to be the concentration of the carbon source. At concentrations of $1-10 \mathrm{mM}$ citrate, spatial heterogeneity in the flow channel was high. While some parts of the channel consisted only of a monolayer of cells, other areas contained large cell clusters. Size heterogeneity was also high. Structures in the biofilms ranged from single cells on the substratum (1-2 $\mu \mathrm{m}$ long) up to microcolonies $200-300 \mu \mathrm{m}$ high. Such size heterogeneity is almost impossible to monitor using a single objective. Finally, the structural variation between channels was high. This variation could in many cases be ascribed to one or more big microcolonies colonizing the inlet of the channel, exhausting the carbon source and leaving the rest of the flow channel essentially as a single layer of cells. Glucose was tried as an alternative to citrate, but no significant differences in biofilm phenotypes were observed. However, by reducing the carbon source concentration to $0.1 \mathrm{mM}$ citrate, the spatial heterogeneity, the size heterogeneity and the structural variation between channels were all drastically reduced. Other important requirements for reproducibility were the following. (1) The history of the cultures used for inoculation should be identical for all strains and identical between different rounds of biofilm experiments. (2) The biofilms should all be grown at the same fixed temperature and temperature shifts should be avoided. If temperature shifts are necessary the time at e.g. $20^{\circ} \mathrm{C}$ should be as short as possible. Temperature shifts should be reproduced in all rounds of the experiments. (3) Bacterial growth upstream of the flow channels (backgrowth) should be removed frequently, for example daily. Backgrowth can significantly alter the structure of the observed biofilm.

\section{Image acquisition, quantification by COMSTAT and selection of variables}

When the experimental conditions have been determined, several rounds of independent biofilm experiments are performed and images are acquired. In the present study, biofilms of $P$. aeruginosa PAO1 and an isogenic rpoS mutant $P$. aeruginosa MW20 were analysed. Three experiment rounds were performed. In each round, each of the two strains was grown in two separate channels. In each channel nine image stacks were acquired at $146 \mathrm{~h}$ after inoculation. Following acquisition, images were quantified by the COMSTAT program, which calculates a wide range of variables describing biofilm structures, such as mean thickness, roughness, surface-to-volume ratio and substratum coverage (for details, see Heydorn et al., 2000). Although it is theoretically possible to use all of the variables calculated by comstat, in most cases a few suffice. The number of selected variables corresponds to the dimensionality of the variable vector used to describe the three-dimensional structure, and to make a subsequent multivariate analysis robust it is important to use a reasonably small number of variables. In most cases it makes sense to simply select the variables on the basis of their biological and physical interpretations in relation to the purpose of the experiments (for details on variable selection see Heydorn et al., 2000). In the present study, only the mean biofilm thickness was analysed. Mean biofilm thickness provides a measure of the spatial size of the biofilm and is the most common variable used in biofilm literature, probably because of its simple interpretation.

\section{Design of an analysis of variance model}

An analysis of variance model is a natural choice when analysing data from experiments similar to the one described here. The general situation is that an experiment gives a univariate (one-dimensional variable) or multivariate (multidimensional variable) continuous response, which depends on a number of registered factors, that in turn may or may not be controlled by the person doing the experiment. The factors may be categorical or continuous, random or fixed (for details see Littell et al., 1996). In cases where the same experimental unit (for example mean thickness) is sampled at different time points, time can be included as an additional factor in an analysis of variance model, or the experiment can be analysed as a 'repeated measurements experiment' (Diggle et al., 1994). The main objective of the present statistical analysis was to verify the reproducibility of experiments, and secondly to distinguish between biofilms of the two $P$. aeruginosa strains after $146 \mathrm{~h}$ of biofilm growth. The factors of the variance model are: bacterial strain (two levels: wildtype and $r$ poS mutant), experimental round (three levels: 1, 2, and 3), and channel (two levels: 1 and 2). Bacterial strain is assumed to be a fixed (or deterministic) factor, whereas experiment round and channel number are assumed to be random factors. They will be denoted $b$, $R$ and $C$, respectively. Finally, the image stacks for each 
combination of bacterial strain, experiment round, and channel are treated as replications. Replication is always assumed to be a random factor. The model thus becomes a variance component model:

$Y_{i j k v}=\mu+b_{i}+R_{j}+B R_{i j}+C(B R)_{k(i j)}+Z_{v(i j k)}$

where $Y_{i j k v}$ is the observed value (e.g. mean thickness) for bacterial strain $i$, experiment round $j$, channel number $k$ and image stack $v . \mu$ is the overall mean value of the experiment, $b_{i}$ is the additional effect of bacterial strain $i, R_{j}$ is the random effect of experiment round $j$, $B R_{i j}$ is a random effect of a possible interaction between bacterial strain $i$ and round $j$, and $C(B R)_{k(i j)}$ is a random effect of channel $k$. Channel $k$ is nested to bacterial strain $i$ and experiment round $j$ because channel $k$ is not unique across bacterial strains and experiment rounds. $Z_{v(i j k)}$ is a random effect of replication. The usual assumptions in this kind of variance model are:

$$
\begin{aligned}
\sum_{i} b_{i}=0, R_{j} \in N\left(0, \sigma_{R}^{2}\right), B R_{i j} \in N\left(0, \sigma_{B R}^{2}\right), \\
C(B R)_{k(i j)} \in N\left(0, \sigma_{C(B R)}^{2}\right), Z_{v(i j k)} \in N\left(0, \sigma^{2}\right)
\end{aligned}
$$

Furthermore, all random effects are assumed to be independent of each other, both within and between effects. If the effect of bacterial strain turns out to be significant (i.e. $b_{i} \neq 0$ ) in the analysis of variance, pairwise $t$-tests can be performed in order to assess which pairs of strains differ from each other.

The analysis of variance model presented here applies to a wider range of scenarios than the simple experiment described. For example, a larger number of strains could be included, more experimental rounds could be performed and more channels per strain could be used.

\section{Analysis of variance model assumptions}

The above model assumptions imply that observations are normally distributed with the mean value $\mu+b_{i}$, and that they have the same variance. The assumption of equal variances is the most important and can be checked numerically, e.g. by Levene's test for homogeneity of variances (Milliken \& Johnson, 1984), or visually, by examining a suitable plot of the data. In some cases a transformation of the data (e.g. by taking the logarithm) or a weighting scheme may help stabilize the variance. The best result is obtained if the weights are proportional to the inverse variance of the observations. However, since the true variance of the observations is rarely known, it is customary to estimate the variance from the experimental data. Figs $2(a)$ and (b) show box-and-whiskers plots (Hoaglin et al., 1991) of the original and log-transformed data, respectively. The original data in Fig. 2(a) clearly display overall differences in the variance of the data, and the variance seems to depend on the mean. Therefore a logarithmic transformation of the data was applied. The logtransformed data generally display a more stable variance (Fig. 2b). Levene's test for equal variances was implemented by PROC GLM in the SAS statistical package
(SAS Institute, 1997) and showed severe differences in variance between the groups. For the original data the $F$ test statistic was 10.5 on $(11,96)$ degrees of freedom, corresponding to a $P$-value $<0 \cdot 0001$, whereas for the log-transformed data the F-statistic was somewhat smaller, at $7 \cdot 4$ on $(11,96)$ degrees of freedom, however still corresponding to a $P$-value $<0 \cdot 0001$. Consequently, both the original data and the log-transformed data displayed severe differences in variance between the groups. It was therefore chosen to perform a weighted analysis of variance, using the inverse estimated variance for each group of data as weights (Fig. 2c).

Another feature of a set of data, which may create problems in an analysis of variance, is the presence of outliers. Outliers are observations that for some reason lie far beyond any usual grouping. Usual causes are gross measurement errors or wrongly attributed data. Outliers may severely invalidate the assumptions of an analysis of variance and thereby lead to wrong conclusions. The presence of outliers in the data can be checked e.g. by Grubb's test for single outliers (International Organization for Standardization, 1995b), or simply by examining a plot of the data. Grubb's test for single outliers was programmed by the authors in a SAS DATA step (SAS Institute, 1990), and a single straggler (significant at the $5 \%$ but not at the $1 \%$ level) was detected for the original, the weighted and the log-transformed data. The observation in question is shown in Fig. 2 as the circle corresponding to bacterial strain 2, experiment round 1 , and channel 2 . The value was checked and found to be correct, so no further action was taken. If an outlier is detected, the experiment protocol should be checked in an attempt to identify the cause of the outlier. Usually it is not possible to correct the kind of experiment described here without redoing an entire experimental round, so in that case we recommend that the statistical analysis is performed both with and without the observation in question.

\section{Examination of experimental reproducibility}

According to ISO standard no. 3534-1 (International Organization for Standardization, 1995a) reproducibility conditions are 'conditions where test results are obtained with the same method on identical test items in different laboratories with different operators using different equipment', whereas repeatability conditions are 'conditions where independent test results are obtained with the same method on identical test items in the same laboratory by the same operator using the same equipment within short intervals of time'. From these definitions, it is not clear whether the variability between experimental rounds in the present experiments should be termed repeatability or reproducibility. We have chosen to use the term reproducibility.

The analysis of variance was performed using PROC MIXED from the SAS statistical package (SAS Institute, 1997). The default estimation technique in PROC MIXED, restricted maximum-likelihood, was used. Reproduci- 

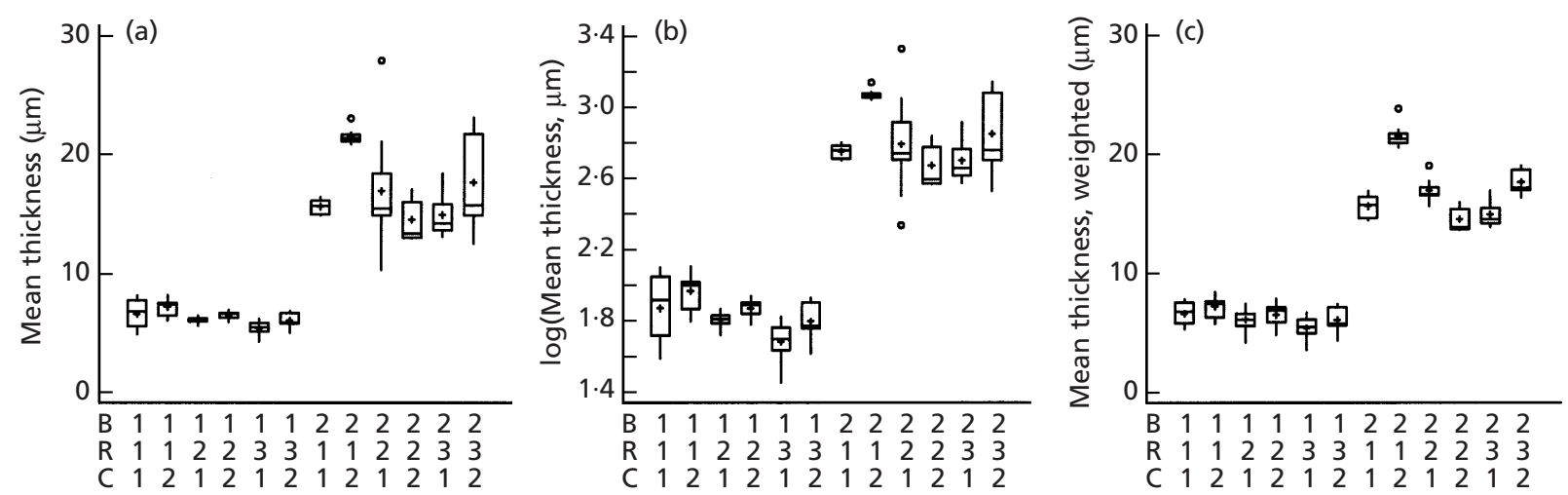

\begin{abstract}
Fig. 2. Box-and-whiskers plots of original data (a), log-transformed data (b) and inverse estimated variance weighted data (c). Each box-and-whiskers symbol represents the 9 image stacks (replications) for each combination of bacterial strain [wild-type (1), MW20 (2)], experiment round $(1,2,3)$ and channel $(1,2) . A^{\prime}+{ }^{\prime}$ represents the mean of the data. The bottom, middle, and top horizontal lines represent the $25 \%$ percentile, the median, and the $75 \%$ percentile of the data, respectively. The distance between the $25 \%$ and $75 \%$ percentile is called the interquartile distance. The vertical lines (whiskers) stretch out to the furthest data point a maximum of 1.5 times the interquartile distance from the $25 \%$ percentile and $75 \%$ percentile, respectively. Any data point outside this is marked as a circle if it is within 3 interquartile distances, or as an asterisk if it is beyond even that. Data points shown as asterisks or circles in plots of this type could be possible outliers (note that there are no data points marked as asterisks in the present graphs).
\end{abstract}

bility was assessed by examining the magnitude of the variance of the effects round $\left(\sigma_{R}^{2}\right)$ and the interaction between bacterial strain and round $\left(\sigma_{B^{*} R}^{2}\right)$. They were estimated at $0.23 \mu \mathrm{m}^{2}\left(\operatorname{se} 1.24 \mu \mathrm{m}^{2}, P\right.$-value $\left.=0.85\right)$ and $0 \cdot 00 \mu \mathrm{m}^{2}$, respectively. Therefore, regarding the variable mean thickness we consider the experiments reproducible. This conclusion is very important in many respects. First, it shows that there are no major variance components that are not considered in the analysis of variance model. Secondly, it demonstrates that the experiments are well controlled, and that other scientists should be able to reproduce the experiments given the same set of experimental conditions. Finally, if reproducibility is expected to be a general property of the present model system, future experiments can be conducted in parallel, i.e. in less time.

The overall $F$-test for the effect of bacterial strain was found to be significant $[P$-value $=0 \cdot 0112$ on $(1,2)$ degrees of freedom], showing that the mean thicknesses of the wild-type and the MW20 biofilms were significantly different. The least-square mean estimates of mean thickness were $6.31 \mu \mathrm{m}(\mathrm{SE} 0.81 \mu \mathrm{m})$ for the wild-type and $16.85 \mu \mathrm{m}($ SE $0.87 \mu \mathrm{m})$ for MW20. The difference in mean thickness between the two strains was estimated at $10.54 \mu \mathrm{m}($ sE $1.12 \mu \mathrm{m})$. The effect of different channels was assessed by the variance component $\sigma_{C\left(B^{*} R\right)}^{2}$, which was estimated at $3.40 \mu \mathrm{m}^{2}$ (SE 1.81, $P$-value $=0.060$ ). Although this is not significant at the $5 \%$ level, it indicates that the channels differ slightly. Finally, the residual or repetition variance $\sigma^{2}$ was estimated at $0.99 \mu^{2}$ (se $0 \cdot 14, P$-value $<0 \cdot 0001$ ). Both un-weighted and weighted analyses of variance were performed on both the original and the log-transformed data. In all four cases the results were very similar. This implies robustness of the analysis of variance.

\section{Biofilm formation of $P$. aeruginosa PAO1 and MW20}

The $r p o S$ gene in $P$. aeruginosa is involved in the general stress response, the accumulation of certain virulence factors, and twitching motility (Jørgensen et al., 1999; Suh et al., 1999). As in E. coli, the stationary-phase sigma factor, RpoS, in $P$. aeruginosa accumulates as cultures enter the stationary phase (Fujita et al., 1994). Recently, it was shown that the lasI-lasR quorumsensing system is necessary for development of $P$. aeruginosa biofilms (Davies et al., 1998). Moreover, the rpoS gene has been shown to be poorly expressed in a $P$. aeruginosa lasR mutant (Latifi et al., 1996), and it has also been shown that RpoS represses the transcription of rhll (Whiteley et al., 2000). However, several questions remain to be answered about the regulatory interactions between RpoS and the quorum-sensing systems in $P$. aeruginosa.

We found in the present investigation that $P$. aeruginosa MW20 formed significantly thicker biofilms than the isogenic parent strain $(6.3$ and $16.9 \mu \mathrm{m}$, respectively). This tendency was not only significant at $146 \mathrm{~h}$, but could already be observed $24 \mathrm{~h}$ after inoculation and throughout the experiment (312 h) (data not shown). Planktonic growth curves of $P$. aeruginosa PAO1 and MW20 in citrate minimal medium showed that the doubling times were virtually identical $(53 \mathrm{~min}$ and 56 min, respectively, at $37^{\circ} \mathrm{C}$ ). Thus, the thicker biofilms formed by MW20 compared to the wild-type do not simply reflect differences in growth rates. Adams \& McLean (1999) found that biofilm cell density of E. coli grown in a modified Robbins device was reduced by $50 \%$ in an rpoS mutant compared to the isogenic parent strain. There are several differences in the role of RpoS between E. coli and P. aeruginosa (Jørgensen et al., 
1999; Suh et al., 1999). It is therefore not surprising that RpoS may play different roles in biofilm development in E. coli and $P$. aeruginosa.

\section{Concluding comments}

Despite the many attempts to quantify biofilm structures, the reproducibility of biofilm experiments has not been addressed until now. We present a general method for quantitative comparison of biofilm structures and assessment of experimental reproducibility between independent biofilm experiments. By using a novel computer program, СОMSTAT, biofilm structures can be quantified and subsequently analysed statistically by an analysis of variance model. In the analysis of variance model presented here, experimental reproducibility was assessed by estimating the magnitude of the variance of the effects round $\left(\sigma_{R}^{2}\right)$ and the interaction between bacterial strain and round $\left(\sigma_{B^{*} R}^{2}\right)$. Securing experimental reproducibility is a necessary prerequisite for conclusions concerning differences between different strains.

\section{ACKNOWLEDGEMENTS}

We thank Kalai Mathee for providing us with flow channels.

\section{REFERENCES}

Adams, J. L. \& McLean, R. J. (1999). Impact of rpoS deletion on Escherichia coli biofilms. Appl Environ Microbiol 65, 4285-4287.

Andersen, J. B., Sternberg, C., Poulsen, L. K., Bjorn, S. P., Givskov, M. \& Molin, S. (1998). New unstable variants of green fluorescent protein for studies of transient gene expression in bacteria. Appl Environ Microbiol 64, 2240-2246.

Christensen, B. B., Sternberg, C., Andersen, J. B., Palmer, R. J., Nielsen, A. T., Givskov, M. \& Molin, S. (1999). Molecular tools for study of biofilm physiology. Methods Enzymol 310, 20-42.

Cunliffe, D., Smart, C. A., Alexander, C. \& Vulfson, E. N. (1999). Bacterial adhesion at synthetic surfaces. Appl Environ Microbiol 65, 4995-5002.

Dalton, H. M., Poulsen, L. K., Halasz, P., Angles, M. L., Goodman, A. E. \& Marshall, K. C. (1994). Substratum-induced morphological changes in a marine bacterium and their relevance to biofilm structure. J Bacteriol 176, 6900-6906.

Davies, D. G., Parsek, M. R., Pearson, J. P., Iglewski, B. H., Costerton, J. W. \& Greenberg, E. P. (1998). The involvement of cell-to-cell signals in the development of a bacterial biofilm. Science 280, 295-298.

Diggle, P. J., Liang, K. Y. \& Zeger, S. L. (1994). Analysis of Longitudinal Data. Oxford: Clarendon Press.

Fujita, M., Tanaka, K., Takahashi, H. \& Amemura, A. (1994). Transcription of the principal sigma-factor genes, $r p o D$ and $r p o S$, in Pseudomonas aeruginosa is controlled according to the growth phase. Mol Microbiol 13, 1071-1077.

Grotenhuis, J. T., Smit, M., Plugge, C. M., Xu, Y. S., van Lammeren, A. A., Stams, A. J. \& Zehnder, A. J. (1991). Bacteriological composition and structure of granular sludge adapted to different substrates. Appl Environ Microbiol 57, 1942-1949.

Heydorn, A., Nielsen, A. T., Hentzer, M., Sternberg, C., Givskov, M., Ersbøll, B. \& Molin, S. (2000). Quantification of biofilm structures by the novel computer program comstat. Microbiology 146, 2395-2407.

Hoaglin, D. C., Mosteller, F. \& Tukey, J. W. (1991). One-way treatment structure in a completely randomized design structure with heterogeneous errors. In Fundamentals of Exploratory Analysis of Variance, pp. 16-28. New York: Wiley-Interscience. Holloway, B. W. (1955). Genetic recombination in Pseudomonas aeruginosa. J Gen Microbiol 13, 572-581.

International Organization for Standardization (1995a). Statistical Methods for Quality Control, vol. 1, Terminology and Symbols, Acceptance Sampling, 4th edn. Geneva, Switzerland: International Organization for Standardization.

International Organization for Standardization (1995b). Statistical methods for quality control, vol. 2, Measurement methods and results, interpretation of statistical data, process control, 4th edn. Geneva, Switzerland: International Organization for Standardization.

Jørgensen, F., Bally, M., Chapon-Herve, V., Michel, G., Lazdunski, A., Williams, P. \& Stewart, G. S. (1999). RpoS-dependent stress tolerance in Pseudomonas aeruginosa. Microbiology 145, 835844.

Korber, D. R., Lawrence, J. R., Hendry, M. J. \& Caldwell, D. E. (1993). Analysis of spatial variability within $\mathrm{Mot}^{+}$and $\mathrm{Mot}^{-}$ Pseudomonas fluorescens biofilms using representative elements. Biofouling 7, 339-358.

Kuehn, M., Hausner, M., Bungartz, H. J., Wagner, M., Wilderer, P. A. \& Wuertz, S. (1998). Automated confocal laser scanning microscopy and semiautomated image processing for analysis of biofilms. Appl Environ Microbiol 64, 4115-4127.

Lanzer, M. \& Bujard, H. (1988). Promoters largely determine the efficiency of repressor action. Proc Natl Acad Sci U S A 85, 8973-8977.

Latifi, A., Foglino, M., Tanaka, K., Williams, P. \& Lazdunski, A. (1996). A hierarchical quorum-sensing cascade in Pseudomonas aeruginosa links the transcriptional activators LasR and RhlR (VsmR) to expression of the stationary-phase sigma factor RpoS. Mol Microbiol 21, 1137-1146.

Lawrence, J. R., Korber, D. R., Hoyle, B. D., Costerton, J. W. \& Caldwell, D. E. (1991). Optical sectioning of microbial biofilms. $J$ Bacteriol 173, 6558-6567.

Littell, R. C., Milliken, G. A., Stroup, W. W. \& Wolfinger, R. D. (1996). SAS System for Mixed Models. Cary, NC: SAS Institute.

de Lorenzo, V., Herrero, M., Jakubzik, U. \& Timmis, K. N. (1990). Mini-Tn5 transposon derivatives for insertion mutagenesis, promoter probing, and chromosomal insertion of cloned DNA in gram-negative eubacteria. J Bacteriol 172, 6568-6572.

Milliken, G. A. \& Johnson, D. E. (1984). Analysis of Messy Data1: Designed Experiments. New York: Van Nostrand Reinhold.

Møller, S., Korber, D. R., Wolfaardt, G. M., Molin, S. \& Caldwell, D. E. (1997). Impact of nutrient composition on a degradative biofilm community. Appl Environ Microbiol 63, 2432-2438.

Murga, R., Stewart, P. S. \& Daly, D. (1995). Quantitative analysis of biofilm thickness variability. Biotechnol Bioeng 45, 503-510.

Nielsen, A. T., Tolker-Nielsen, T., Barken, K. B. \& Molin, S. (2000). Role of commensal relationships on the spatial structure of a surface-attached microbial consortium. Environ Microbiol 2, 59-68.

O'Toole, G. A. \& Kolter, R. (1998). Initiation of biofilm formation in Pseudomonas fluorescens WCS365 proceeds via multiple, convergent signalling pathways: a genetic analysis. Mol Microbiol 28, 449-461.

Palmer, R. J., Jr (1999). Microscopy flowcells: perfusion chambers for real-time study of biofilms. Methods Enzymol 310, 160-166.

Picioreanu, C., Van Loosdrecht, M. C. M. \& Heijnen, J. J. (1998). 
Mathematical modeling of biofilm structure with a hybrid differential-discrete cellular automaton approach. Biotechnol Bioeng 58, 101-116.

SAS Institute (1990). SAS Language: Reference, Version 6, 1st edn. Cary, NC: SAS Institute.

SAS Institute (1997). Changes and Enhancements through Release 6.12. Cary, NC: SAS Institute.

Stoodley, P., Boyle, J. D., DeBeer, D. \& Lappin-Scott, H. M. (1999a). Evolving perspectives of biofilm structure. Biofouling 14, 75-90.

Stoodley, P., Lewandowski, Z., Boyle, J. D. \& Lappin-Scott, H. M. (1999b). Structural deformation of bacterial biofilms caused by short-term fluctuations in fluid shear: an in situ investigation of biofilm rheology. Biotechnol Bioeng 65, 83-92.

Suh, S. J., Silo-Suh, L., Woods, D. E., Hassett, D. J., West, S. E. \& Ohman, D. E. (1999). Effect of rpoS mutation on the stress response and expression of virulence factors in Pseudomonas aeruginosa. J Bacteriol 181, 3890-3897.

Van Loosdrecht, M. C. M., Eikelboom, D., Gjaltema, A., Mulder, A., Tijhuis, L. \& Heijnen, J. J. (1995). Biofilm structures. Water Sci Technol 32, 35-43.

Whiteley, M., Parsek, M. R. \& Greenberg, E. P. (2000). Regulation of quorum sensing by RpoS in Pseudomonas aeruginosa. I Bacteriol 182, 4356-4360.

Wimpenny, J. W. T. \& Colasanti, R. (1997). A unifying hypothesis for the structure of microbial biofilms based on cellular automaton models. FEMS Microbiol Ecol 22, 1-16.

Wolfaardt, G. M., Lawrence, J. R., Robarts, R. D., Caldwell, S. J. \& Caldwell, D. E. (1994). Multicellular organization in a degradative biofilm community. Appl Environ Microbiol 60, 434-446.

Received 12 June 2000; revised 14 July 2000; accepted 21 July 2000. 